\title{
Computed Tomography-Based Tumor Heterogeneity Analysis Reveals Differences in a Cohort with Advanced Pancreatic Carcinoma under Palliative Chemotherapy
}

\author{
Jochen Paul Steinacker ${ }^{a}$ Nora Steinacker-Stanescu ${ }^{a}$ Thomas Ettrich ${ }^{b}$ \\ Marko Kornmann ${ }^{c}$ Katharina Kneer ${ }^{d}$ Ambros Beer ${ }^{d}$ Meinrad Beer ${ }^{a}$ \\ Stefan Andreas Schmidt ${ }^{a}$

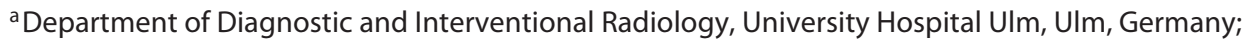 \\ ${ }^{b}$ Department for Internal Medicine I, University Hospital Ulm, Ulm, Germany; ${ }^{\mathrm{C} D e p a r t m e n t ~ f o r ~ G e n e r a l ~ a n d ~}$

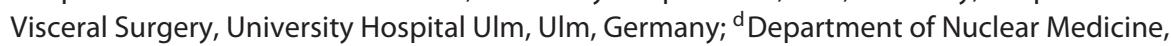 \\ University Hospital UIm, Ulm, Germany
}

\section{Keywords \\ Pancreatic cancer · Tumor heterogeneity · Computed tomography · Image analysis · Image biomarker}

\begin{abstract}
Purpose: Imaging in pancreatic cancer is a challenge, especially regarding therapy response evaluation. Tumor size, attenuation, and perfusion are widely used as parameters for computed tomography (CT) examinations, but are often limited due to blurry tumor borders and missing qualitative parameters. To improve monitoring of therapy response, we tested a new CT-based approach of tumor heterogeneity feature analysis. Methods: A total of 13 patients with pancreatic adenocarcinoma undergoing abdominal CT according to standard as baseline imaging with clinical follow-up and imaging (median time span 64 days) under systematic therapy (FOLFIRINOX/gemcitabine) were retrospectively analyzed. Progression was defined as new lesions and local tumor spread. Tumor heterogeneity analysis was performed using mintLesion ${ }^{\circledR}$. Seven different image features referring to image heterogeneity were analyzed. Statistical analysis was performed with Spearman's rank correlation and MannWhitney U test. Results: During follow-up, tumor volume did
\end{abstract}

not significantly change between our groups with overall progression (local and systemic) and progression-free patients $(p=0.661)$. Mean positivity of pixel values were significantly higher in patients without progression compared to patients with progression $(p=0.030)$. There was a significant negative correlation between changes in kurtosis and time to local tumor spread ( $p=0.008$ ) or systemic progression ( $p=0.017)$. Conclusions: Results suggest that analysis of tumor heterogeneity might provide valuable information from routine-acquired images regarding therapy response evaluation. This might help adjusting therapy regimes and could be easily integrated in clinical workflows. Furthermore, this procedure might possibly predict therapy response and, hence could lead the way to find a potential marker for progression-free survival.

(c) 2020 S. Karger AG, Basel

\section{Introduction}

Despite advances in oncologic cancer treatment, pancreatic ductal adenocarcinoma cancer is among the tumor entities with the worst prognosis, with a high incidence in both men and women [1]. 
Fig. 1. Flowchart showing number of baseline and follow-up examinations. * Abort due to therapy intolerance.

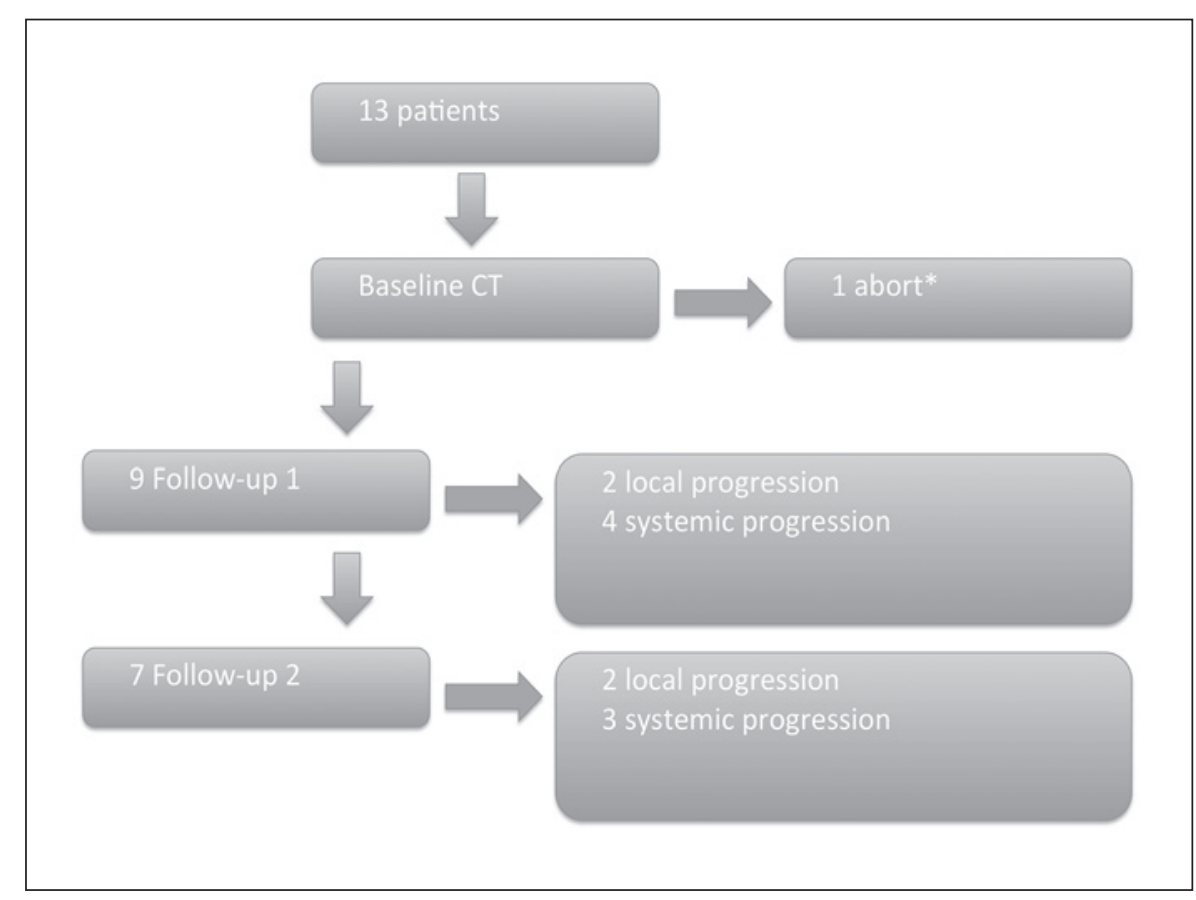

A variety of drugs have been applied for palliative therapy with limited activity like gemcitabine [2]. Progress has been achieved with novel combined chemotherapy protocols like FOLFIRINOX or NAB-paclitaxel, which are of importance in the context of palliative and neoadjuvant therapy [3]. However, while these chemotherapy protocols can be highly active, usually only a subset of patients profits from these rather aggressive treatments. Thus, early response evaluation based on imaging is of high relevance in this scenario. However, imaging in pancreatic cancer is challenging, especially regarding therapy response evaluation. While tumor size changes and attenuation are widely used as measurable parameters for baseline and follow-up examinations, they are also limited by blurry tumor borders and missing quantitative parameters. Efforts have been made to improve the accuracy in identifying patients with local progression, for instance by applying PET/CT analysis to obtain a higher sensitivity for local tumor spread [4]. Computed tomography $(\mathrm{CT})$ remains as an essential imaging tool besides endoscopy ultrasound and MRI [5].

In the past decades, tumor heterogeneity has been identified as a major characteristic in the approach of decoding tumor biology, e.g., by the means of genomic profiling and molecular medicine [6]. Also imaging features are increasingly used to analyze tumor heterogeneity, including radiomics analysis and machine learning approaches [7-10]. In this respect, CT-based texture analysis is a promising technique based on the analysis of the distribution of grey-level values using histogram analysis. There have been promising results from studies in this context on a variety of tumor enti- ties, CT texture characteristics (including entropy, mean positivity of pixels [MPP], standard deviation [SD]) showed correlation with histologic findings, tumor grading as well as outcome in patients with renal cell carcinoma $[11,12]$.

Based on these promising results, we tested a new CTbased approach of tumor heterogeneity feature analysis for evaluating therapy response of patients with advanced pancreatic cancer. The objective of this study was the correlation of imaging features derived from CT-images with local and systemic tumor progression in follow-up examinations under systemic therapy. Our hypothesis was the existence of differences in texture analysis between responders and nonresponders and consecutively the prediction of progression. Since patients suffering from pancreatic adenocarcinoma with an advanced tumor stage unfortunately show rapid progress and have a very limited prognosis, we focused on comparing stable versus progressive disease only, differentiating local tumor progression and systemic progression with new metastatic lesions.

\section{Material and Methods}

Retrospective analysis included the imaging datasets of 13 patients with pancreatic adenocarcinoma in the time span between August 2011 and April 2016, who were included in our study having undergone systemic therapy according to standard and showing homogeneous imaging data with baseline and follow-up CT examinations in a defined portal venous contrast media phase. Figure 1 summarizes the process of patient study inclusion. Informed consent was obtained from the patients prior to the examinations. 


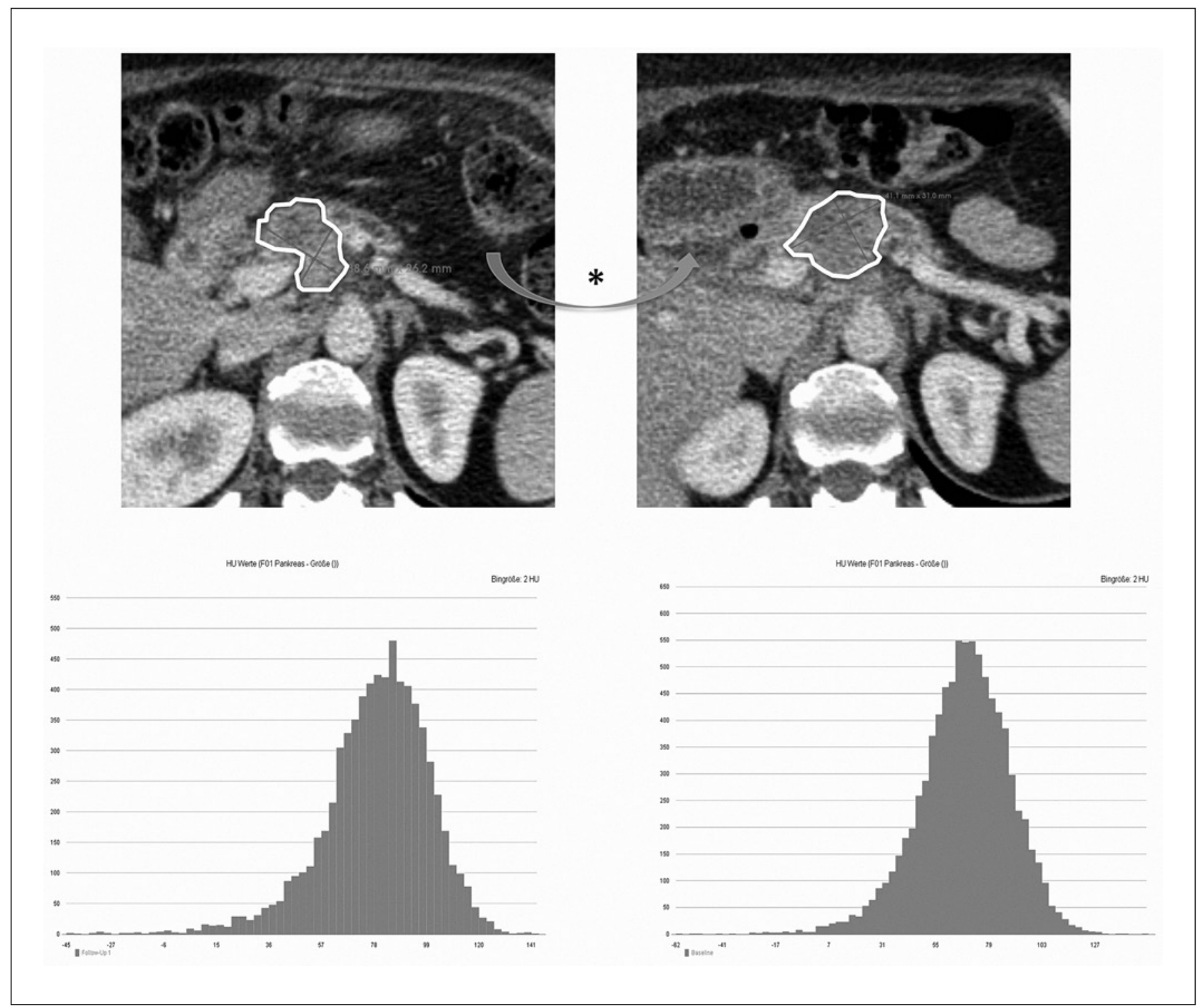

Fig. 2. Images of a 54-year-old female patient with an adenocarcinoma of the head of the pancreas. Upper left panel: baseline CT, white surrounding indicating ROI with crossed lines indicating tumor diameter and tumor volume at $12.2 \mathrm{~cm}^{3}$. Upper right panel: at follow-up, local progression with progressive perivascular tu- mor spread and tumor volume at $14.2 \mathrm{~cm}^{3}$ are shown. * Arrow indicating induction systemic therapy with FOLFIRINOX. Lower panels: histograms with distribution of $\mathrm{HU}$ on the $\mathrm{x}$-axis against corresponding number of pixels within the ROI.
The diagnosis of pancreatic ductal adenocarcinoma was histopathologically confirmed in all patients. Inclusion criteria were advanced stages with unresectable tumors, scheduled for a standardized systemic therapy. Systemic therapy included the combination of folinic acid, fluorouracil, irinotecan, oxaliplation (FOLFIRINOX; $n=7)$ and gemcitabine $(n=6)$. Exclusion criteria were tumor surgery and substantial imaging artifacts due to foreign material, e.g., metal stents or abortion of systemic therapy due to intolerance.

The patients underwent CT of the abdomen as baseline imaging with clinical follow-up and imaging (median time span 64 days) under systematic therapy according to standard. The CT protocol included an arterial contrast phase of the upper abdomen and a portal venous phase of the whole abdomen as well as a thoracic CT as staging examination; all $31 \mathrm{CT}$ examinations included intravenous contrast media injection (Ultravist ${ }^{\circledR} 370$ ) in weight-adopted dose.
The examinations were carried out with a Philips Brilliance 64 channel scanner and a Philips iCT scanner. Imaging parameters were helical scanning with a slice thickness of $1 \mathrm{~mm}$, tube current and amplitude were in a range of $135-336 \mathrm{mAs}$ and $100-120 \mathrm{kVp}$, including dose modulation techniques. Image reconstruction was performed with a soft tissue kernel, the reconstructed image slice thickness was $4 \mathrm{~mm}$.

Progression was defined as new tumor lesions, which occurred in 6 patients, and local tumor spread, which was detected in 3 patients with progressive local tumor masses and progressive vessel infiltration. Two patients showed local and systemic tumor progression.

CT images were retrieved from the archive of the institutional picture archiving and communication system (PACS) to a clinical workstation. Tumor heterogeneity analysis was performed using mintLesion ${ }^{\circledR}$. Tumor separation was performed in 


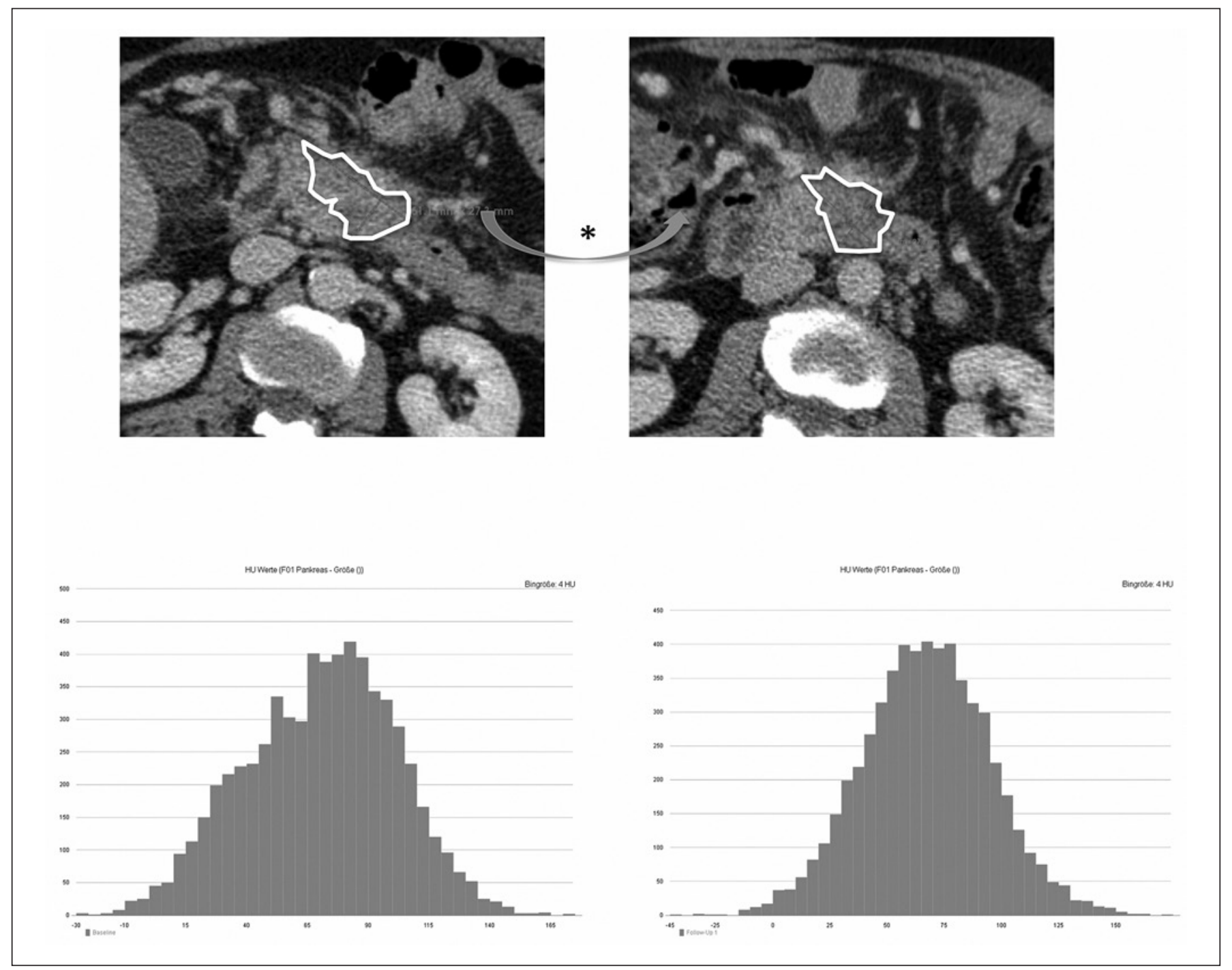

Fig. 3. Images of a 52-year-old male patient with adenocarcinoma of the body of the pancreas and synchronic metastasis of peritoneum and liver. Upper left panel: baseline CT, white surrounding indicating ROI with crossed lines indicating tumor diameter and tumor volume at $18.7 \mathrm{~cm}^{3}$. Upper right panel: at follow-up, stable

a semiautomatic process, a trained radiologist defined the region of interest (ROI) with manual correction of the automatically marked tumor borders, sparing anatomic structures inside the tumor borders, such as vessels, to avoid errors in the measurements.

Heterogeneity analysis of CT images based on grey-value distribution was performed on CT images of 13 patients with advanced tumor disease with baseline examination and follow-ups with a standardized protocol. Seven different image characteristics including tumor volume, entropy, kurtosis, MPP, skewness, uniformity of distribution of positive pixels (UPP), and uniformity of pixels were validated, leading to a dataset of 231 histograms.

Statistical analysis was performed with Spearman's rank correlation and Mann-Whitney U test, assuming normal distribution according to the Shapiro-Wilk test. We calculated the standard error of mean for our results, Student's $t$ test was applied and statistical significance was assumed with $p$ values $<0.05$.

tumor disease, tumor volume at $18.7 \mathrm{~cm}^{3} . *$ Arrow indicating induction systemic therapy with FOLFIRINOX. Lower panels: histograms with distribution of $\mathrm{HU}$ on the $\mathrm{x}$-axis against corresponding number of pixels within the ROI.

\section{Results}

\section{Patient Groups}

Representative examples of histograms with the distribution of pixels with different Hounsfield units (HU) are shown in Figures 2 and 3. It shows a case (Fig. 2) with local tumor progression under therapy and a case (Fig. 3) with stable disease during follow-up, defined by visual criteria of local tumor progression, e.g., vessel invasion or macroscopic tumor spread.

\section{Tumor Size during Follow-Up}

Two groups were segregated, one containing patients with local $(n=3)$ or systemic $(n=6)$ tumor progression, the other one with patients showing stable dis- 


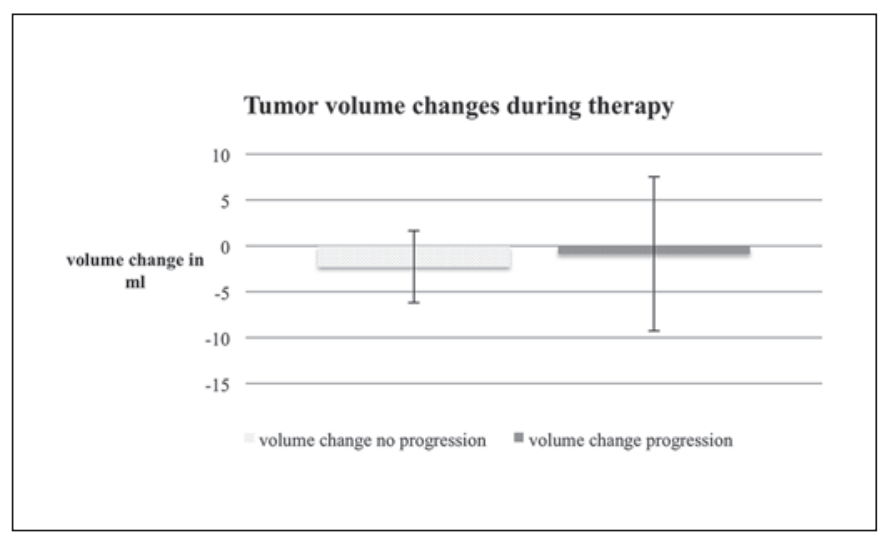

Fig. 4. Graphic showing changes of the mean value of tumor volume. Comparison of tumor size at follow-up examinations with tumor size at baseline examination. Left bar showing changes of tumor volume in the group of progression-free patients, right bar showing mean changes of tumor volume in the group with progression, each with standard error of mean. No significant difference was found. $p$ value $=0.66$.

ease $(n=4)$. During follow-up, tumor volume did not significantly change between our groups with overall progression (local and systemic) with an average tumor volume change of $-0.87 \pm 8.39 \mathrm{~mL}$ standard error of mean (SEM) and progression-free patients $(p=0.661)$ with an average tumor volume change of $-2.25 \pm 3.93$ mL (SEM) (Fig. 4).

\section{Tumor Heterogeneity during Follow-Up and Correlations}

Analysis of imaging parameters referring to tumor heterogeneity revealed significant differences in our two patient subpopulations. MPP values were significantly higher in patients without progression compared to patients with progression ( $p=0.030)$ (Fig. 5). Mean values at baseline examination were $69.0 \pm 3.92 \mathrm{HU}$ (SEM), dropping to a mean value of $63.1 \pm 2.65 \mathrm{HU}$ (SEM) in patients without progression and to lower values of $59.9 \pm$ $4.06 \mathrm{HU}$ (SEM) in average for patients with progressive disease.

There was a significant negative correlation between changes in kurtosis and the time until local tumor spread $(p=0.008)$ or systemic progression $(p=0.017)$ (Fig. 6). The time span of tumor progression reached from 35 to 334 days.

Spearman's rank correlation revealed no significant correlation between time of progression and tumor volume ( $p=0.164)$, entropy $(p=0.235)$, MPP differences $(p$ $=0.832)$, differences in skewness $(p=0.272)$, UPP changes $(p=0.247)$, and changes in uniformity of pixels $(p=$ $0.247)$.

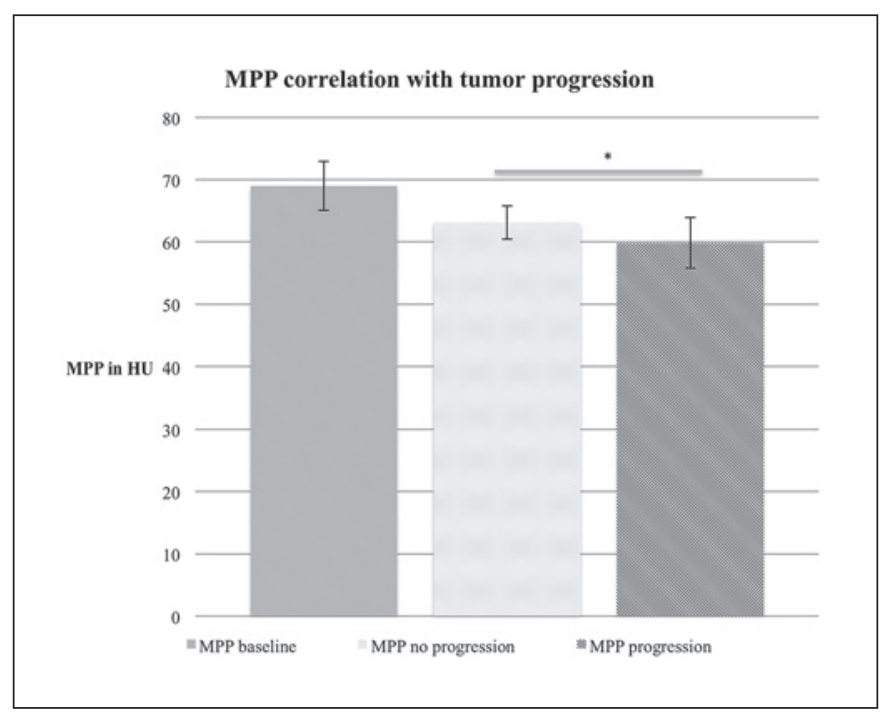

Fig. 5. Graph showing values for MPP in HU at baseline and followup examinations, black bars indicating standard error of mean. ${ }^{*} p$ value at 0.03 for MPP differences between groups without tumor progression (middle) and with tumor progression (right).

\section{Discussion}

Our results are concordant with the results of other groups which demonstrated possible advantages of heterogeneity analysis of grey level value distribution compared to changes in tumor volume [13], which showed no significant differences in our patient subpopulations with or without overall tumor progression. This might be due to blurry tumor borders and consecutive hard-to-define areas of tumor infiltration, which aggravate the assessment of therapy response by mere optical criteria, which might be an imaging correlate for formerly described perineural and angiolymphatic tumor infiltration [14].

We were able to identify significant differences of the MPP in the tumor volume between our patient subpopulations, which showed higher levels of MPP in the progression-free group. MPP values have been in the focus of other studies in the past which analyzed correlations between MPP values and histological subtypes of tumors and the outcome, where significant correlations could be identified, for example, in the comparison of different kidney tumors [11]. Our results are supported by other study outcomes, which lately identified hypoattenuation in pancreatic cancer detected in CT images as a potential characteristic of more aggressive tumors and as a robust procedure to differentiate between tumor tissue and normal pancreatic tissue $[12,15,16]$.

Significant correlations were found for other image features derived from histogram analysis, such as entropy correlated with the overall survival within patients with hepatic metastases from colorectal carcinoma [17], whereas this marker showed no significant correlation 
Fig. 6. Graph showing correlation of changes in kurtosis (x-axis) and time until progression (local and systemic) in days ( $\mathrm{y}$ axis).

\section{Correlation of time until progress and differences in kurtosis until progress}

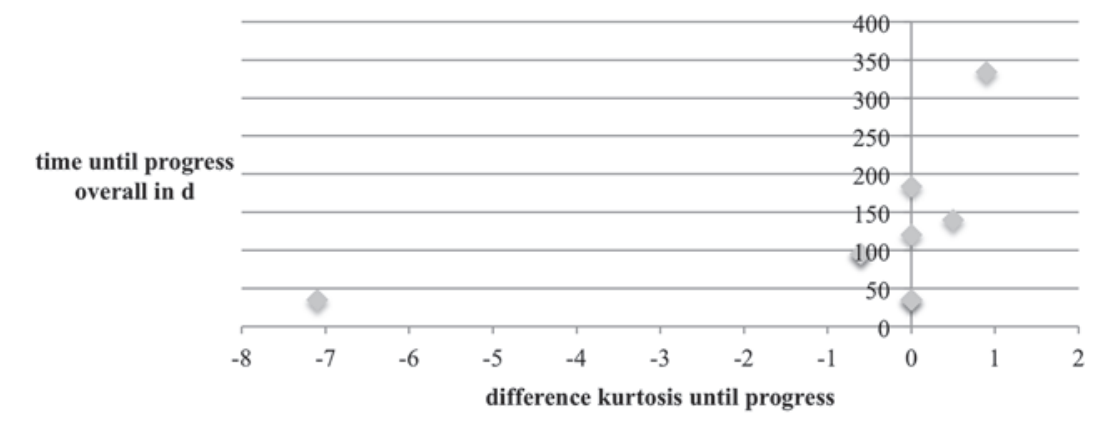

with either of our subpopulations. This might be due to the limited number of patients included in our study and has to be further investigated in a wider approach with a higher number of subjects.

We identified a negative correlation between changes in kurtosis and the time until local and systemic progression. Kurtosis is a parameter which tells us about the tails of the distribution of pixels in our histogram and indirectly provides information about the peakedness of the distribution, which has been discussed in other studies coping with imaging tumor heterogeneity [18], for instance in the context of hepatocellular cancer [19] or lung cancer [20]. The higher the changes in kurtosis were, the shorter the time span became until tumor progression was detected in our patient subpopulations. Higher values of kurtosis represent a higher heterogeneity of the grey-level value distribution with heavier tails of the distribution, which means the more homogeneous the tumors appeared under therapy, the longer it took until progression was detected. This finding might be one fragment in detecting imaging biomarkers regarding risk stratification and therapy response monitoring as discussed for other tumor entities in the past [21-24].

\section{Limitations}

The patient population included in the current study is small. However, the recruitment of "naive" patients (no prior surgical or conservative treatment) is challenging, as most patients received treatment prior to admission to the tertiary centers such as a university hospital. Additionally, only one reader performed tumor heterogeneity analysis, thus intra- or interobserver variability analyses could not be done. However, the main aim of the present study is a proof-of-principle concept regarding the feasibility of texture analysis in the context of pancreatic carcinoma. Due to the aggressiveness of this tumor type, the included follow-up time span was limited. In summary, further studies with a larger cohort of patients are warranted to determine the real benefits and potential of tumor heterogeneity analysis in pancreatic adenocarcinoma. To obtain larger cohorts, multi-center studies might be helpful; thus differences in technical equipment, such as different scanners, and variables in image reconstruction, for example, different reconstruction kernels might be challenging, since homogeneous datasets are essential for robust heterogeneity analysis $[25,26]$.

Despite these limitations, tumor heterogeneity analysis might become a powerful tool in monitoring therapy response, predicting therapy response and hence might be regarded as a potential future imaging biomarker in patients with advanced pancreatic adenocarcinoma.

In conclusion, we were able to show that imaging features depicting tumor heterogeneity, derived from CT datasets acquired during clinical routine, can show differences in responders and nonresponders undergoing systemic chemotherapy for advanced pancreatic adenocarcinoma, which has to be further investigated in future studies.

\section{Acknowledgements}

The authors thank the Ulm University Center for Translational Imaging MoMAN for its support.

\section{Statement of Ethics}

The Ethics Committee provided approval to this study (application No. 459/17). Informed consent was obtained from all individual participants included in the study.

\section{Disclosure Statement}

The authors have no conflicts of interest to declare. 


\section{Funding Sources}

This research received no specific grant from any funding agency in the public, commercial, or not-for-profit sectors.

\section{Author Contributions}

Jochen Paul Steinacker, Nora Steinacker-Stanescu, Katharina Kneer, Ambros Beer, Meinrad Beer, Stefan Andreas Schmidt: Evaluation of CT images and performance of semiautomatic tumor outlineage, statistical analysis of histogram datasets.

Thomas Ettrich, Marko Kornmann: experts for clinical management of advanced tumor diseases, consultation for multidisciplinary approach.

\section{References}

1 Leitlinienprogramm Onkologie der Arbeitsgemeinschaft der Wissenschaftlichen Medizinischen Fachgesellschaften V. (AWMF), der Deutschen Krebsgesellschaft e.V. (DKG) und der Deutschen Krebshilfe. S3-Leitinie zum exokrinen Pankreaskarzinom, Stand 03/2017. Available at: https://www.leitlinienprogramm-onkologie.de/fileadmin/user_upload/Downloads/Leitlinien/Pankreaskarzinom/LL_Pankreas_OL_Langversion.pdf (accessed January 5th 2020).

2 Kipps E, Young K, Starling N. Liposomal irinotecan in gemcitabine-refractory metastatic pancreatic cancer: efficacy, safety and place in therapy. Ther Adv Med Oncol. 2017 Mar; 9(3):159-70.

3 Pompa TA, Morano WF, Jeurkar C, Li H, Soundararajan S, Poli J, et al. Complete Response after Treatment with Neoadjuvant Chemoradiation with Prolonged Chemotherapy for Locally Advanced, Unresectable Adenocarcinoma of the Pancreas. Case Rep Oncol Med. 2017;2017:7834702.

4 Toesca DA, Pollom EL, Poullos PD, Flynt L, Cui Y, Quon A, et al. Assessing local progression after stereotactic body radiation therapy for unresectable pancreatic adenocarcinoma: CT versus PET. Pract Radiat Oncol. 2017 Mar - Apr;7(2):120-5.

5 Dallongeville A, Corno L, Silvera S, BoulayColetta I, Zins M. Initial Diagnosis and Staging of Pancreatic Cancer Including Main Differentials. Semin Ultrasound CT MR. 2019 Dec;40(6):436-68.

6 Esparza-López J, Escobar-Arriaga E, SotoGermes S, Ibarra-Sánchez MJ. Breast Cancer Intra-Tumor Heterogeneity: One Tumor, Different Entities. Rev Invest Clin. 2017 MarApr;69(2):66-76.

7 Bian Y, Guo S, Jiang H, Gao S, Shao C, Cao K, et al. Relationship Between Radiomics and Risk of Lymph Node Metastasis in Pancreatic Ductal Adenocarcinoma. Pancreas. 2019 Oct; 48(9):1195-203.

8 Gu D, Hu Y, Ding H, Wei J, Chen K, Liu H, et al. CT radiomics may predict the grade of pancreatic neuroendocrine tumors: a multicenter study. Eur Radiol. 2019 Dec;29(12): 6880-90.
9 Yang J, Guo X, Ou X, Zhang W, Ma X. Discrimination of Pancreatic Serous Cystadenomas From Mucinous Cystadenomas With CT Textural Features: Based on Machine Learning. Front Oncol. 2019 Jun;9:494.

10 Yamashita R, Perrin T, Chakraborty J, Chou JF, Horvat N, Koszalka MA, et al. Radiomic feature reproducibility in contrast-enhanced $\mathrm{CT}$ of the pancreas is affected by variabilities in scan parameters and manual segmentation. Eur Radiol. 2020 Jan;30(1):195-205.

11 Lubner MG, Stabo N, Abel EJ, Del Rio AM, Pickhardt PJ. CT Textural Analysis of Large Primary Renal Cell Carcinomas: Pretreatment Tumor Heterogeneity Correlates With Histologic Findings and Clinical Outcomes. AJR Am J Roentgenol. 2016 Jul;207(1):96105.

12 Chu LC, Park S, Kawamoto S, Fouladi DF, Shayesteh S, Zinreich ES, et al. Utility of CT Radiomics Features in Differentiation of Pancreatic Ductal Adenocarcinoma From Normal Pancreatic Tissue. AJR Am J Roentgenol. 2019 Aug;213(2):349-57.

13 Goh V, Ganeshan B, Nathan P, Juttla JK, Vinayan A, Miles KA. Assessment of response to tyrosine kinase inhibitors in metastatic renal cell cancer: CT texture as a predictive biomarker. Radiology. 2011 Oct;261(1):165-71.

14 Schorn S, Demir IE, Haller B, Scheufele F, Reyes CM, Tieftrunk E, et al. The influence of neural invasion on survival and tumor recurrence in pancreatic ductal adenocarcinoma A systematic review and meta-analysis. Surg Oncol. 2017 Mar;26(1):105-15.

15 Cassinotto C, Chong J, Zogopoulos G, Reinhold C, Chiche L, Lafourcade JP, et al. Resectable pancreatic adenocarcinoma: role of CT quantitative imaging biomarkers for predicting pathology and patient outcomes. Eur J Radiol. 2017 May;90:152-8.

16 Eilaghi A, Baig S, Zhang Y, Zhang J, Karanicolas P, Gallinger S, et al. CT texture features are associated with overall survival in pancreatic ductal adenocarcinoma - a quantitative analysis. BMC Med Imaging. 2017 Jun;17(1): 38.

17 Lubner MG, Stabo N, Lubner SJ, del Rio AM, Song C, Halberg RB, et al. CT textural analysis of hepatic metastatic colorectal cancer: pretreatment tumor heterogeneity correlates with pathology and clinical outcomes. Abdom Imaging. 2015 Oct;40(7):2331-7.
18 Davnall F, Yip CS, Ljungqvist G, Selmi M, Ng F, Sanghera B, et al. Assessment of tumor heterogeneity: an emerging imaging tool for clinical practice? Insights Imaging. 2012 Dec; 3(6):573-89.

19 Hectors SJ, Wagner M, Bane O, Besa C, Lewis S, Remark R, et al. Quantification of hepatocellular carcinoma heterogeneity with multiparametric magnetic resonance imaging. Sci Rep. 2017 May;7(1):2452.

20 Yoon SH, Park CM, Park SJ, Yoon JH, Hahn S, Goo JM. Tumor Heterogeneity in Lung Cancer: Assessment with Dynamic Contrastenhanced MR Imaging. Radiology. 2016 Sep; 280(3):940-8.

21 Feng C, Lu F, Shen Y, Li A, Yu H, Tang H, et al. Tumor heterogeneity in gastrointestinal stromal tumors of the small bowel: volumetric CT texture analysis as a potential biomarker for risk stratification. Cancer Imaging. 2018 Dec;18(1):46.

22 Andersen IR, Thorup K, Andersen MB, Olesen R, Mortensen FV, Nielsen DT, et al. Texture in the monitoring of regorafenib therapy in patients with colorectal liver metastases. Acta Radiol. 2019 Sep;60(9):1084-93.

23 Digumarthy SR, Padole AM, Gullo RL, Sequist LV, Kalra MK. Can CT radiomic analysis in NSCLC predict histology and EGFR mutation status? Medicine (Baltimore). 2019 Jan; 98(1):e13963.

24 Nasief H, Zheng C, Schott D, Hall W, Tsai S, Erickson B, et al. A machine learning based delta-radiomics process for early prediction of treatment response of pancreatic cancer. NPJ Precis Oncol. 2019 Oct;3(1):25.

25 Gardin I, Grégoire V, Gibon D, Kirisli H, Pasquier D, Thariat J, et al. Radiomics: principles and radiotherapy applications. Crit Rev Oncol Hematol. 2019 Jun;138:44-50.

26 Korn RL, Rahmanuddin S, Borazanci E. Use of Precision Imaging in the Evaluation of Pancreas Cancer. Cancer Treat Res. 2019;178: 209-36. 